\title{
Image Feature Extraction of Numbers and Letters Using Ma- trix Segmentation
}

\author{
F. Budiman ${ }^{1}$, E. Sugiarto² \\ Department of Computer Science, University of Dian Nuswantoro, Semarang, Indonesia \\ ${ }^{1}$ ORCID: 00oo-0oo2-8552-6778, fikri.budiman@dsn.dinus.ac.id \\ 2 ORCID: 0000-0002-7354-0652, edi.sugiarto@dsn.dinus.ac.id
}

\begin{abstract}
Classification in recognizing image of letters and numbers is useful to recognize vehicle license plates. This study aims to maximize classification accuracy value of feature extraction method using matrix segmentation. The dataset consists of 300 vehicle license plate images which have 36 classifications, 26 classes for A-Z letters image, and 10 classes for 0-9 numbers image. The research stages carried out to maximize the results of the classification using multiclass SVM-RBF nonlinear are: preparing region image of interest, image enhancement, image segmentation of letters and numbers, determining the best $\mathrm{n}$ value for $\mathrm{n} \times \mathrm{n}$ matrix segmentation, calculating total points of each segment as feature value, and determining the best value for $\mathrm{C}$ and $\gamma$ as the value of $\mathrm{RBF}$ kernel parameter. The result of this study shows a maximum value of $92 \%$ classification accuracy using $n=5, \gamma=0.8$, and $C=15$.
\end{abstract}

Keywords: Matrix segmentation, feature extraction, vehicle license plate, accuracy, Support Vector Machine.

\section{Introduction}

This study is conducted to gain a maximum classification accuracy of the image of letters and numbers. A high accuracy value is required for feature extraction stages to produce good image features. The method of feature extraction in this research is performed by using image matrix segmentation. The images of numbers and letters used as training and testing dataset are obtained from the result of segmentation of vehicle lisence plates. This research which is included as a research in the field of Vehicle License Plate Recognition (VLPR) is aimed to recognize character images in recognizing the combination of letters and numbers stamped in vehicle lisence plates. VLCR research has been done a lot as it is significantly urgent and necessary to ease in identifying vehicle owners. There are three important discussions in recognizing vehicle license plates: License Plate Detection, Character Segmentation and Character Recognition [1][2]. Recognition of the features in each number and letter on the license plate is an important step in VLPR study since it influences the thoroughness in producing maximum accuracy and the speed in processing overall recognition system [3].

This study focuses on the method of segmentation and feature extraction using matrix segmentation to produce a character features dataset of letters and numbers on lisence plates. Feature extraction highly depends on the results of image segmentation, and the feature dataset from feature extraction significantly influences the value of accuracy in recognizing images using classification algorithms. Accordingly, the better the image as the region of interest produced in segmentation process is, the easier the feature extraction process is to the image, and the produced feature dataset will increase the classification accuracy value. A precise feature extraction method in producing feature datasets is a fundamental part of classification since feature datasets produced from good feature extraction can maximize the accuracy of classification results [3][4]. 
Non-linear multi-class classification algorithm used in study is Support Vector Machine (SVM) with a Radial Based Function (RBF) kernel. The results of previous studies said that the classification accuracy value with SVM has a better success rate than the Artificial Neural Network (ANN) [5] and Backpropagation Neural Network (BNN) [6]. ANN algorithm is a combination of statistics and knowledge trees which have a higher computational complexity than SVM. The results of non-linear classification with BNN can not be consistent because there are differences in the results of the model of decision boundary margins between classes on each use of different feature test data, while the SVM can provide the same class decision limit model (global optima). In general, the use of Neural Networks in the classification will experience overfitting problems for large datasets. Non-linear SVM is chosen to maximize the value of classification accuracy by handling overfitting with soft margins. This is conducted by replacing each dot product of test feature with non-linear kernel function matrix. Consequently, it is necessary to determine the best kernel for available datasets in non-linear multiclass classification with SVM method. RBF kernel is used in similar studies because it is recommended to gain maximum non-linear classification results for a new dataset [7]-[11]. Classification using SVM for image features with high data dimensions can significantly improve classification accuracy [9][12]. The use of SVM in classification is advantageous as it does not involve all training vector dataset of image feature in forming hyperplane and margin as class separators; only the contributing vector dataset of image feature (support vector) is used in the forming hyperplane and margin. SVM has shown a high accuracy value to classify lisence plates and other images in previous studies. In previous studies, the authors have optimized the use of multi-grade SVM in image recognition using Geometric Decorative Motifs [13]. Lihong Zheng and Xiangjian He from University of Technology Sydney have conducted a research on the use of Support Vector Machine method for character recognition of Australian vehicle license plates [14]. The purposes of their study is to compare two kernel methods, Linear Kernel and Radial Basis Kernel Function, while the multi-class method used in their study is "One Against All" method. Kumar and Subin conducted a study on vehicle lisence plates recognition using Support Vector Machine method [15], and the scope of the study is to detect the location of lisence plates, to segment the character of vehicle lisence plates and to test the accuracy of the method using multi-class SVM and RBF kernel.

Such efforts have been conducted in the previous VLPR research to develop feature extraction to maximize the value of classification accuracy using multi-class SVM. Andrej and Nikola, in his license plate recognition study, increased the accuracy (83\%) as a result of multiclass SVM classification with Compressive Sensing based feature extraction [16]. Compressive Sensing was used to minimize the use of sample data or training feature dataset. In addition, to improve the quality of feature dataset in Fet Xie, et al all [17], feature extraction is developed to produce features consisting of 3 characteristics: Vertical Traverse Density (VTD), Horizontal Traverse Density (HTD), and the distance from the left edge to the first white pixel. In other license plate detection studies [18] [19], classification using Multiclass SVM is conducted to increase the accuracy value by feature extraction using Histogram of Oriented Gradient (HOG). Feature dataset from the extracted feature vector of each normalized candidate is used as training and testing dataset on Multiclass SVM classification with RBF kernel [20]. In other studies, feature dataset which is produced by Enhanced geometrical feature topological analysis (eGFTA) is used to improve the accuracy value of SVM classification result [21].

In increasing the accuracy value of multiclass SVM classification result, the authors in this study, create a feature extraction method in generating feature dataset by simplifying the determination of feature values. Moreover, image matrix segmentation is used to count total pixels per segment $n$th line $\mathrm{m}$ th column as feature vector value for each character of letter or number. The methods were as follow: 1 . transformation of scanned image to ROI Image; 2. image enhancement to eliminate unnecessary noise in ROI image; 3. skeleton process for each image segmentation result; 4 . finding the value of height and width in the image of letters/numbers which have undergone image skeletonization process; 5 . finding the right matrix segmentation; 6. finding total pixels as a feature stored in each segment on the matrix. 
The method for producing letter and number feature values carried out in this study has a simpler computational complexity than the methods used in previous studies. Feature extraction is generally carried out by statistical and spectral methods. In the statistical method in determining the parameter features performed based on the distribution of gray level frequency, the segmentation matrix is used to determine the proximity between pixels as a function of orientation and spatial distance in digital images. In the Spectral method characteristic parameters are calculated by transforming the spatial domain image into a signal domain, in this method the matrix is used to describe the transformation scheme [22]. The use of Matrix Based Feature Extraction Algorithms is applied to the principal component analysis (PCA) and linear discriminant analysis (LDA) methods, to form a matrix covariance of the matrix of the mean values of images in looking for eigenvalues [23]. Searching for eigenvalue requires more time for images with high resolution, for example, if images with a resolution of 256x256 will produce a vector with a dimension value of 65526 , so with a large dimension, the calculation of covariance matrix in producing eigenvalues will take longer. In this study, there is a process simplicity, because the segmentation matrix always uses a $5 \times 5$ size and produces a 25-dimensional vector as a feature, regardless of the magnitude of the image resolution. The use of matrix segmentation is based on a survey conducted by Mingqiang et. al. [24], in the survey, a comparative study was carried out on 40 techniques for extraction of shape-base features. The results of the survey have concluded that the ease of feature extraction processes with low computational complexity and more efficient processes, is using the Shape Matrix which is a Spatial Interrelation Feature method. The Shape Matrix is described by the MxN matrix to represent the shape region of each image.

\section{Research Method}

The data used in this study are digital images obtained by scanning vehicle license plates in Indonesia (figure 1) with black background and white characters of letters/numbers. The image dataset consists of the vehicle license plate is 300 (three hundred) samples taken under random conditions and lighting on the research objects and the time of taking/scanning the image is between 10:00 to 15:00. License plate [ in Indonesia is regulated based on the Article 68 Paragraph 4 Law Number 22 year 2009 on Traffic and Public Transportation. The law explains that license plate number must meet the requirements of shape, size, material, color, and method of installation. However, it does not contain the standard rules for the use of font type. Thus, the character of letters and numbers on lisence plate may vary greatly. This diversity in fact creates its own complexity in generating feature dataset.

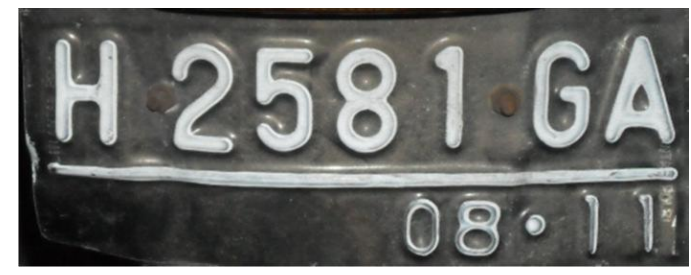

Fig. 1. Image Example of Scanned Lisence Plate

\subsection{Binary Image and Region of Interest}

This research focuses on how to do segmentation process of the scanned image, and how feature extraction method is applied to the segmented image with a variety of font types for each letter or number. Moreover, feature extraction results in a feature dataset for numbers $0-9$ and letters A-Z which will be used for training and testing of non-linear multi-class SVM classification using RBF kernel and one against-all method. Finding Region of interest (ROI) from the scanned images is conducted before the stages in segmentation process. The pseudo code to generate ROI can be described as follows: 


\section{Description}

[line,column] $\longleftarrow$ size(imgIn);
StartingPoint $\longleftarrow$ int16(line/3);

\section{Implementation}

Binarization $\{$ RGB to binary image with pixel value o or $1 \rightarrow$ figure 2.$\}$

for $\mathrm{i}=$ StartingPoint:-1:1

total $1 \longleftarrow 0$;

for $j=1$ :column

$\operatorname{if}(\operatorname{img} \operatorname{In}(i, j)>0)$

total $1 \longleftarrow$ total $1+1$;

end

end

\{if total pixel value 1 less than $1 / 8$ column $\}$

if $($ total $1<($ int16 $(($ column/4)/2) $))$

end

break; \{repetation end \}

end

The result of binarization and ROI is seen in Figure 2 and 3.

(A)

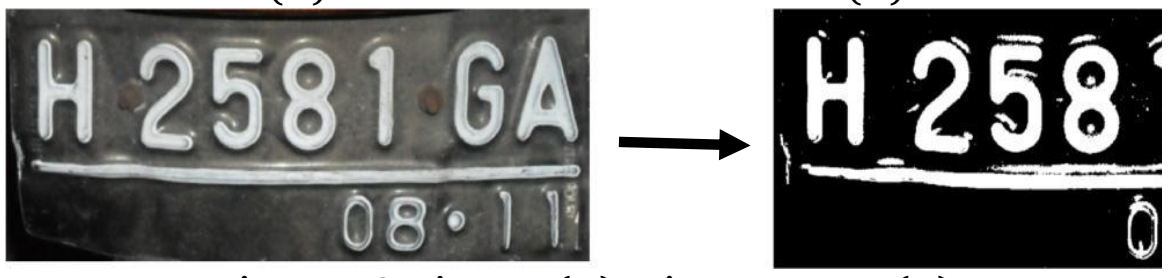

Fig. 2. RGB image (A), Binary Image (B)
(B)

\section{H2581 GA \\ 06.1 1

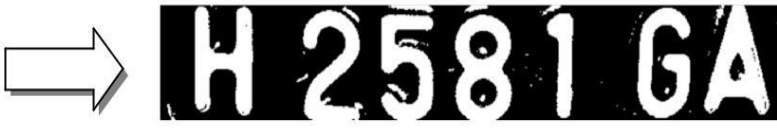

Fig. 3. Result of transformation of scanned images to ROI Image

\subsection{Image Enhancement}

The next step prior to segmentation is to do image enhancement on ROI image to eliminate unnecessary noise. This study used matlab function: bwareaopen ( ). Denoising process aims to simplify segmentation process in separating letters and numbers on a lisence plate from its black background. This process can be seen in this following figure 4 .

(A)

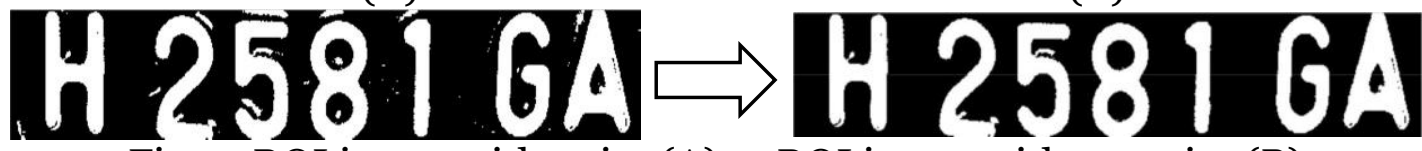

Fig. 4. ROI image with noise (A) to ROI image without noise (B) 


\subsection{Segmentation Method}

ROI images without noise will become input of letters (A-Z) and numbers (1-9) using segmentation process. Segmentation method proposed in this study used this following algorithm:

1. Mark the connected pixels.

2. Conduct looping or repetation starting from 1 to the marked maximum pixel marked.

3. Find the appropriate pixel label, if it is found, insert it in the row and column matrix.

4. Save the image from min row to max row and min column to max column.

5. If it is not the end of the loop, skip to command 3 , and if is the end of the loop, then the loop has finished.

The pseudo code of the algorithm can be seen in this following:

\section{Description}

Imgn=imageInput \{image input image after preprocessing $\}$;

[L Ne] = bwlabel(imgn);

\section{Implementation}

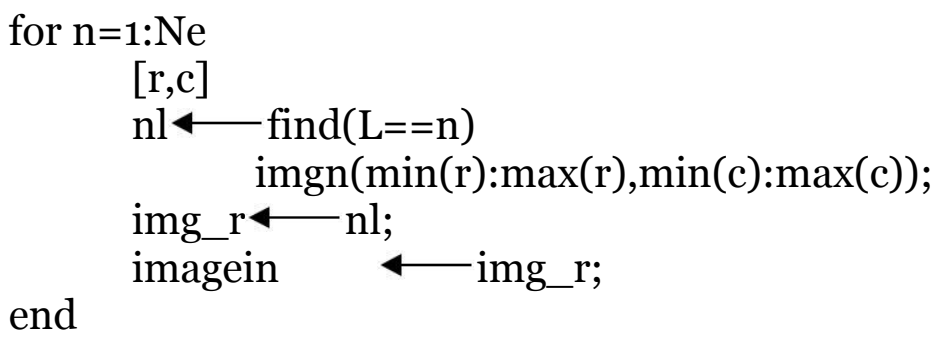

The results of the segmentation method above is in the form of image pieces of letters and numbers existing in ROI image. Moreover, the image resulted from the segmentation consisting of 4 numbers and 3 letters is as shown in Figure 5 below.

(A)

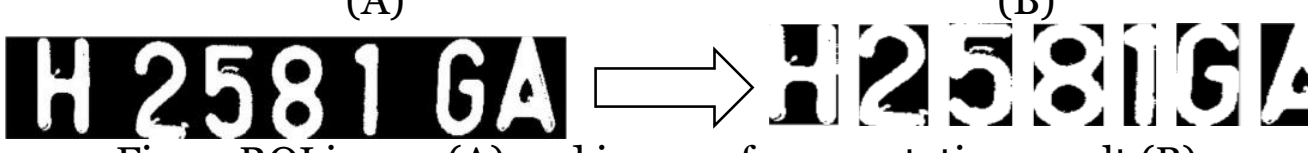

Fig. 5. ROI image (A) and image of segmentation result (B)

As the input is in the form of lisence plate images which vary in the type or shape of each letter and number, the method in this research used 5 variations for each image letter or figure from segmentation results and these will be used as training image input. Accordingly, the number of image in datasets is 180 images which were used in trained. This number of images comes from the number of lisence plate number images (o-9) x $5=50$ images and lisence plate letter images $(A-Z) \times 5=130$. Figure 6 shows the examples of training image datasets that will be extracted to produce training feature datasets: 


\begin{tabular}{|c|c|c|c|c|c|}
\hline $\begin{array}{l}\text { letter / } \\
\text { number } \\
\text { ( class ) }\end{array}$ & Image 1 & Image 2 & Image 3 & Image 4 & Image 5 \\
\hline $\bar{A}$ & & & & & \\
\hline$\overline{\bar{B}}$ & & 8 & & .1 & \\
\hline C & & & & & \\
\hline D & & & & & \\
\hline $\mathbf{E}$ & $\equiv$ & 푼 & Fid & 를 & \\
\hline 5 & TI & & & 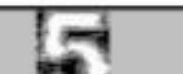 & \\
\hline 6 & [8] & 8 & 6 & (ด) & \\
\hline 7 & 87 & 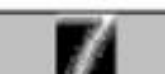 & 7 & $7 d$ & \\
\hline 8 & [क] & 8 & 8 & [?] & 8 \\
\hline 9 & & 9 & 9 & $\theta$ & $Q$ \\
\hline
\end{tabular}

Fig. 6. Example of Training Image Dataset

\subsection{Method of Extraction Feature}

Feature extraction method used in this study is by using image matrix segmentation to find the features in each letter (A-Z) and number (o-9) that will be used as training and testing feature dataset in classification. The followings are the stages of feature extraction:

1. Finding the height and width value of each character which has been transformed in skeletonization process. In matlab, skeletonization process can use bwmorph () function. Skeleton process causes the letters or numbers become like a skeleton as seen in Figure 7. To simplify feature extraction process, skeleton image is standardized to $40 \mathrm{x} 40$ pixels.

2.

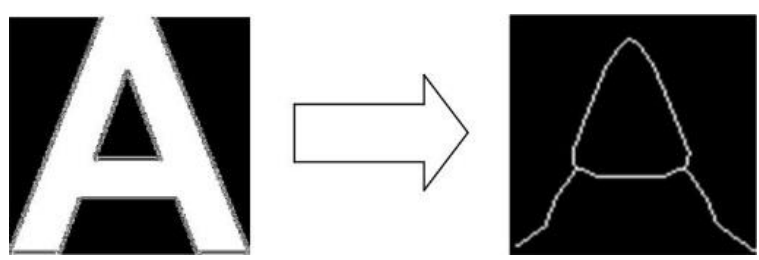

Fig. 7. Result of Skeletonization Process

The height and width of the letters or numbers on the image resulted from skeletonization process is then calculated. Furthermore, it is mapped into the matrix $\mathrm{n} \times \mathrm{n}$ based on the height and width of the image. Figure 8 shows an example of skeleton image which is mapped into 5 × 5 matrix segmentation.

3. Counting total pixels per matrix segment as a feature. Thus, each image number or letter has $\mathrm{n} \times \mathrm{n}$ total features stored in the feature vector. In Figure 8, letter A image is mapped 
to a $5 \times 5$ matrix segmentation possessing 25 matrix segments of which each segment is counted by total number of pixels and total pixels in each segment shows its feature value. The examples of feature extraction resulted by counting total pixels of each matrix segment as feature values are shown in table 1.

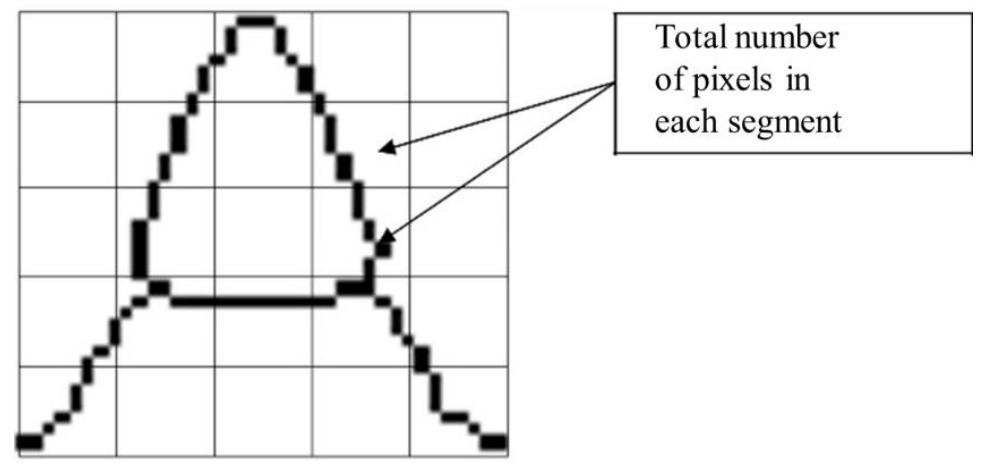

Fig. 8. 5x5 Image Matrix Segmentation

The following is the pseudo code calculation of total pixels per matrix segment on $\mathrm{n} \times \mathrm{n}$ matrix segmentation:

\section{Description}

Line $\longleftarrow$ the number of image lines

Column $\longleftarrow$ the number of image columns

Average pixel per line $\longleftarrow$ int16(line/n)

Ave pixel per col $\longleftarrow$ int16(column/n)

Total Pixel $\longleftarrow$ [ ];

\section{Implementation}

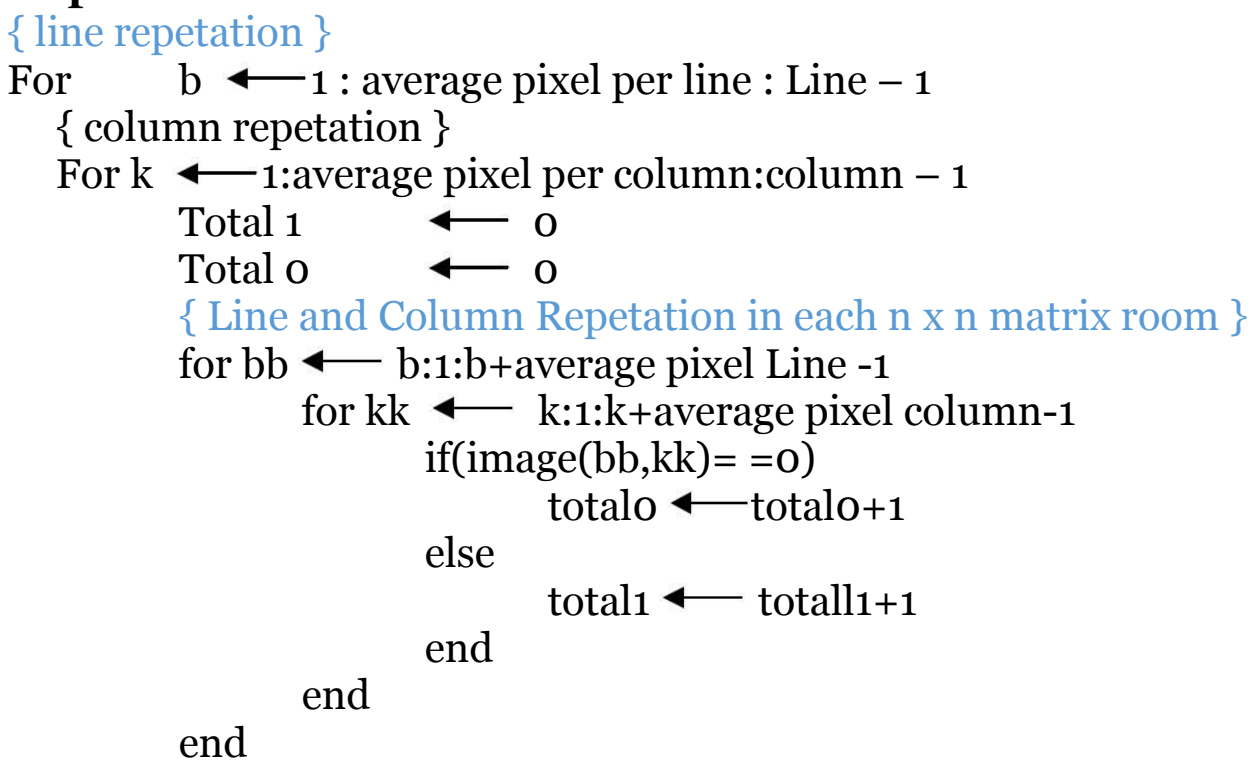

Total Pixel $1 \longleftarrow$ Vertcat (TotalPix1,Total 1/10)

end

end

output $\longleftarrow$ [TotalPix1]; 
Table 1. Feature Vector from Feature extraction for 5 image types of letter A

\begin{tabular}{|c|c|c|c|c|c|}
\hline Class & $\begin{array}{c}\text { Feature Vector } \\
\text { of } \\
\text { Image } 1\end{array}$ & $\begin{array}{c}\text { Feature Vector } \\
\text { of } \\
\text { Image } 2\end{array}$ & $\begin{array}{c}\text { Feature Vec- } \\
\text { tor of } \\
\text { Image } 3\end{array}$ & $\begin{array}{c}\text { Feature Vec- } \\
\text { tor of } \\
\text { Image } 4\end{array}$ & $\begin{array}{c}\text { Feature Vec- } \\
\text { tor of } \\
\text { Image } 5\end{array}$ \\
\hline \multirow{25}{*}{$\mathbf{A}$} & $\mathrm{O}$ & $\mathrm{O}$ & $\mathrm{O}$ & $\mathrm{O}$ & $\mathrm{O}$ \\
\hline & $\mathrm{O}$ & $\mathrm{O}$ & $\mathrm{O}$ & 0 & 0 \\
\hline & 0.70 & 1.20 & 0.90 & 0.50 & O \\
\hline & $\mathrm{O}$ & O & $\mathrm{O}$ & O & 0.30 \\
\hline & $\mathrm{O}$ & $\mathrm{O}$ & $\mathrm{O}$ & $\mathrm{O}$ & 0.80 \\
\hline & O & O & $\mathrm{O}$ & $\mathrm{O}$ & $\mathrm{O}$ \\
\hline & 0.70 & 0.80 & 0.70 & 0.60 & $\mathrm{O}$ \\
\hline & 0.40 & 1.10 & 0.90 & 0.50 & 1.00 \\
\hline & 0.50 & 0.70 & $\mathrm{O}$ & 0.50 & 0.70 \\
\hline & $\mathrm{O}$ & $\mathrm{O}$ & $\mathrm{O}$ & $\mathrm{O}$ & 0.80000 \\
\hline & $\mathrm{O}$ & $\mathrm{O}$ & $\mathrm{O}$ & $\mathrm{O}$ & $\mathrm{O}$ \\
\hline & 0.80 & 0.80 & 0.80 & 0.80 & 0.10 \\
\hline & $\mathrm{O}$ & $\mathrm{O}$ & 0.20 & $\mathrm{O}$ & 0.70 \\
\hline & 0.80 & 0.80 & 0.60 & 0.80 & $\mathrm{O}$ \\
\hline & $\mathrm{O}$ & $\mathrm{O}$ & $\mathrm{O}$ & 0 & 0.80 \\
\hline & 0.10 & 0.40 & 0.20 & $\mathrm{O}$ & $\mathrm{O}$ \\
\hline & 1.00 & 0.90 & 1.10 & 1.20 & 0.80 \\
\hline & 0.80 & 0.80 & 0.80 & 0.80 & 0.80 \\
\hline & 1.10 & 1.00 & 1.00 & 1.10 & 0.80 \\
\hline & 0.10 & 0.30 & $\mathrm{O}$ & $\mathrm{O}$ & 0.90 \\
\hline & 0.80 & 0.90 & 1.30 & 0.90 & 0.90 \\
\hline & $\mathrm{O}$ & 0 & 0.40 & 1.0 & 0.10 \\
\hline & $\mathrm{O}$ & 0 & $\mathrm{O}$ & $\mathrm{O}$ & $\mathrm{O}$ \\
\hline & $\mathrm{O}$ & 0 & 1.20 & 0.20 & $\mathrm{O}$ \\
\hline & 0.90 & 0.80 & 0.80 & 0.70 & 0.60 \\
\hline
\end{tabular}

\section{Results and Analysis}

Feature extraction testing is conducted in this study with value ranging from $n=2$ ( 4 features) to $n=8$ (64 features) for $n \times n$ matrix segmentation. The datasets with 300 images of vehicle lisence plate which are randomly divided into 200 images as training dataset and 100 images as testing dataset. This test is to find the best $\mathrm{n}$ value, so $\mathrm{n} \mathrm{x} \mathrm{n}$ matrix can create the best feature vector to get maximum classification accuracy value. Segmentation matrix used in this study is $5 \times 5(n=5)$ consisting of 25 segments. The number of each segment is calculated and total pixel of each segment means one feature value. Thus, each image has 25 features stored in the feature vector. The use $n=5$ is based on the trial test with value from $n=2$ to $n=8$ in feature extraction method with image matrix segmentation. The trial shows the value of classification result decreases if the number of features declines on the feature vector $<25(n<5)$ and the number of features increases at feature vector $>25(n>5)$. From the previous authors research [4], Wavelet decomposition levels analysis for feature extraction with SVM classification, it shows that the use of the number of features in feature vector obtained from wavelet decomposition influences the results of SVM classification. consequently, it is necessary to use the right level of decomposition. This case is similar in this study, where the number of features in the feature vector resulted from feature extraction using matrix segmentation affects the results of SVM classification. In conclusion, it requires testing with some $n$ values, and the results of the trials (table 2) show that maximum accuracy value occur at $n=5$. 
Table 2. Effects of $n$ Value on Accuracy Value with SVM-RBF C $=15$ and $\gamma=0.8$.

\begin{tabular}{|l|l|l|l|}
\hline$N$ Value & $\begin{array}{l}\text { Matrix segmenta- } \\
\text { tion }\end{array}$ & $\begin{array}{l}\text { Number of seg- } \\
\text { ment/feature }\end{array}$ & $\begin{array}{l}\text { Over All Accu- } \\
\text { racy (AC) }\end{array}$ \\
\hline 2 & $2 \times 2$ & 4 & 0,58 \\
\hline 3 & $3 \times 3$ & 9 & 0.64 \\
\hline 4 & $4 \times 4$ & 16 & 0.83 \\
\hline 5 & $5 \times 5$ & 25 & 0.92 \\
\hline 6 & $6 \times 6$ & 36 & 0.79 \\
\hline 7 & $7 \times 7$ & 49 & 0,70 \\
\hline 8 & $8 \times 8$ & 64 & 0,62 \\
\hline
\end{tabular}

Non-linear multi-class classification in this study consists of 36 classes consisting of 26 classes for the image of letter $\mathrm{A}-\mathrm{Z}$ and 10 classes for numbers $0-9$. Confusion matrix $(\mathrm{AC}=(\mathrm{TP}+$ $\mathrm{FP}) /(\mathrm{TP}+\mathrm{FP}+\mathrm{TN}+\mathrm{FN}))$ is used to calculate the accuracy value for measuring the success ratio of the classification result. The right value of $\mathrm{RBF}$ kernel parameter cost function / $\mathrm{C}$ and gamma $/ \gamma$ is required in the classification using non-linear multi-class SVM-RBF with the one-against-all method. The correct value of parameters $\mathrm{C}$ and $\gamma$ can maximize the function of classification (hyperplane) and can adjust the equilibrium of margin distance (class +1 and -1) with the right hyperplane [9]. From feature dataset of 100 images resulted from feature extraction features with $5 \times 5(n=5)$ matrix segmentation, classification is conducted with the highest accuracy value of 0.92 (90\%) using parameter values $\mathrm{C}=15$ and $\gamma=0.8$ as seen in table 3 .

Table 3. Measurement of Accuracy Level

\begin{tabular}{|c|c|c|c|c|c|c|}
\hline \multicolumn{2}{|c|}{ SVM Parameter } & $\begin{array}{l}\text { True } \\
\text { Positive } \\
(T P)\end{array}$ & $\begin{array}{l}\text { False } \\
\text { Positive } \\
\text { (FP) }\end{array}$ & $\begin{array}{l}\text { True } \\
\text { Negative } \\
\text { (TN) }\end{array}$ & $\begin{array}{l}\text { False } \\
\text { Negative } \\
(F N)\end{array}$ & $\begin{array}{l}\text { Over All } \\
\text { Accuracy } \\
(A C)\end{array}$ \\
\hline \multirow{5}{*}{$\gamma=0.4$} & $\mathrm{C}=\mathrm{O}$ & o & 93 & 7 & $\mathrm{O}$ & 0,07 \\
\hline & $\mathrm{C}=5$ & 32 & 61 & 7 & 0 & 0,39 \\
\hline & $\mathrm{C}=10$ & 32 & 61 & 7 & $\mathrm{O}$ & 0.39 \\
\hline & $C=15$ & 35 & 58 & 7 & 0 & 0,42 \\
\hline & $C=20$ & 34 & 59 & 7 & o & 0,41 \\
\hline \multirow{5}{*}{$\gamma=0.6$} & $\mathrm{C}=\mathrm{O}$ & o & 93 & 7 & $\mathrm{O}$ & 0,07 \\
\hline & $C=5$ & 69 & 24 & 7 & $\mathrm{O}$ & 0,76 \\
\hline & $\mathrm{C}=10$ & 70 & 23 & 7 & 0 & 0,77 \\
\hline & $\mathrm{C}=15$ & 72 & 21 & 7 & 0 & 0,79 \\
\hline & $C=20$ & 71 & 22 & 7 & o & 0,78 \\
\hline \multirow{5}{*}{$y=0.8$} & $\mathrm{C}=\mathrm{O}$ & 0 & 93 & 7 & 0 & 0,07 \\
\hline & $\mathrm{C}=5$ & 81 & 12 & 7 & $\mathrm{O}$ & 0,88 \\
\hline & $C=10$ & 82 & 11 & 7 & $\mathrm{O}$ & 0,89 \\
\hline & $C=15$ & 85 & 8 & 7 & 0 & 0,92 \\
\hline & $C=20$ & 81 & 12 & 7 & 0 & 0,88 \\
\hline \multirow{5}{*}{$\gamma=1.0$} & $\mathrm{C}=\mathrm{O}$ & o & 93 & 7 & $\mathrm{O}$ & 0,07 \\
\hline & $\mathrm{C}=5$ & 76 & 17 & 7 & 0 & 0,83 \\
\hline & $C=10$ & 77 & 16 & 7 & $\mathrm{O}$ & 0,84 \\
\hline & $\mathrm{C}=15$ & 78 & 15 & 7 & 0 & 0,85 \\
\hline & $C=20$ & 76 & 17 & 7 & 0 & 0,83 \\
\hline \multirow[t]{2}{*}{$\gamma=1.2$} & $\mathrm{C}=\mathrm{O}$ & o & 93 & 7 & $\mathrm{O}$ & 0,07 \\
\hline & $\mathrm{C}=5$ & 72 & 21 & 7 & $\mathrm{o}$ & 0,79 \\
\hline
\end{tabular}




\begin{tabular}{|l|l|l|l|l|l|}
$\mathrm{C}=10$ & 74 & 19 & 7 & $\mathrm{O}$ & 0,81 \\
\hline $\mathrm{C}=15$ & 74 & 19 & 7 & $\mathrm{O}$ & 0,81 \\
\hline $\mathrm{C}=20$ & 73 & 20 & 7 & 0 & 0,8 \\
\hline
\end{tabular}

Table 2 describes that $\mathrm{C}$ value from o to 15 causes the accuracy value increase, and $\mathrm{C}$ value more than 15 causes the accuracy value decrease. This is due to the fact that an increase in variance at $\mathrm{C}>15$ causes over fitting. Value of parameter $\gamma>0.8$ increases the bias so that misclassification increases due to under fitting, and there is an increase of variance in value of parameter $\gamma<0.8$, thus there are more misclassification because of over fitting.

\section{Conclusion}

Testing and training feature dataset resulted from feature extraction methods with $5 \times 5$ ( $\mathrm{n}=$ 5) image matrix segmentation is a feature dataset with the best $n$ value for classification of letters and numbers images using non-linear multi-class SVM-RBF method with value of parameter $\mathrm{C}=15$ and $\gamma=0.8$. Image segmentation method of vehicle lisence plate and feature extraction using image matrix segmentation in this study is very easy to apply, and is successfully applied for multi-class classification with a total of 36 classes, 26 classes for A-Z letter images and 10 classes for 0-9 number images with $92 \%$ accuracy value. The feature dataset produced by feature extraction method in this study with more classification classes needs further research to test based on processing time and the result of classification accuracy value.

Matrix segmentation with $\mathrm{n}=5$ results in 25 features on each image feature vector. However, it still requires a trial to add feature value to the features vector of letters and numbers images which are in the form of centroid values and the value of letters' height and width.

\section{References}

1. Shuang Qiao, "Research of Improving the Accuracy of License Plate Character Segmentation", Fifth International Conference on Frontier of Computer Science Technology, 2010.

2. Choudhury A. Rahman, Wael Badawy,"A Real Time Vehicle License Plate Recognition System”, Proceedings of the IEEE Conference on Advance Video and Signal Based Surveillance (AVSS'O3), 2003.

3. Xiaojuan Ma, Renlong Pan, Lin Wang, "License Plate Character Recognition Based on Gaussian-Hermite Moments", 2010 Second International Workshop on Education Technology and Computer Science, Guizhou Key Lab of Pattern Recognition \& Intelligent Control Guizhou University for Nationalities Guiyang, China, 2010

4. Budiman F., Suhendra A., Agushinta D., Tarigan A., "Wavelet Decomposition Levels Analysis For Indonesia Traditional Batik Classification”. Journal of Theoretical \& Applied Information Technology, 92(2):389-394. 2016.

5. Ahmad M. Sarhan, "Wavelet-based Feature Extraction For DNA Microarray Classification”, Springer Science+Business Media B.V. Artif Intell Rev, 39:237-249. 2013.

6. Yuan, Qing-Ni, Lu, Jian, Huang, Haisong, dan Pan, Weiji, 2014, "Research of Batik Image Classification Based On Support Vector Machine", Computer Modelling \& New Technologies 18(12B) 193-196, 2014.

7. Hofmann, Martin. "Support Vector Machine-Kernel and The Kernel Trick", Bamberg University, 2006.

8. Hsu, Chih-Wei, Chang, Chih-Chung, Lin Chih-Jen, "A Practice Guide to Support Vector Classification". Department of Computer Science, National Taiwan University, 2010.

9. Renukadevi, N.T., Thangaraj, P., "Performance Evaluation Of Svm-Rbf Kernel For Medical Image Classification”, Global Journal of Computer Science and Technology Graphics \& Vision, vol.13 issue 4, Global Journal Inc, USA, 2013. 
10. Rosales-Perez, Alejandro, Escalante, Hugo Jair, Gonzales, Jesus A., Reyes-Garcia, Carlos A., "Bias And Variance Optimization For Svms Model Selection", Proceedings of the Twenty-Sixth International Florida Artificial Intelligence Research Society Conference, 2013.

11. Budiman F., Suhendra A., Agushinta D., \& Tarigan A., "Determination Of SVM-RBF Kernel Space Parameter To Optimize Accuracy Value Of Indonesian Batik Images Classification", Journal of Computer Science. 13(11):590-599, 2017.

12. Azhar, Ryfial,Tuwohingide, Desmin, Kamudi, Dasrit, Sarimuddin., dan Suciati, Nanink,"Batik Image Classification Using SIFT Feature Extraction, Bag of Features and Support Vector Machine”, Procedia Computer Science 72(2015)24-30, Elsevier, 2015.

13. Budiman F. "SVM-RBF Parameters Testing Optimization Using Cross Validation and Grid Search to Improve Multiclass Classification”, Scientific Visualization, vol.11(1): 80-90, 2019.

14. Lihong Zheng, Xiangjian He,"Number Plate Recognition Based on Support Vector Machine", Proceeding of the IEEE International Conference on Video and Signal Based Surveillance, 2006.

15. Kumar Parasuraman, "SVM Based License Plate Recognition System”, IEEE International Conference on Computational Intelligence and Computing Research, 2010.

16. Jokic A, Vukovic N. "License Plate Recognition with Compressive Sensing Based Feature Extraction”. arXiv preprint arXiv:1902.05386. 2019.

17. Xie F, Zhang M., Zhao J., Yang J., Liu Y., Yuan X. A., Robust License Plate Detection and Character Recognition Algorithm Based on a Combined Feature Extraction Model and BPNN. Journal of Advanced Transportation. 2018.

18. Astawa IN, Caturbawa IG, Sajayasa IM, Atmaja IM. "Detection of License Plate using Sliding Window, Histogram of Oriented Gradient, and Support Vector Machines Method”. In Journal of Physics: Conference Series 2018 Jan (Vol. 953, No. 1, p. 012062). IOP Publishing.

19. Negri P. "A MATLAB SMO Implementation to Train a SVM Classifier: Application to Multi-Style License Plate Numbers Recognition”. Image Processing On Line. 22;8:51-70. 2018.

20. Kumar Parasuraman, "SVM Based License Plate Recognition System”, IEEE International Conference on Computational Intelligence and Computing Research, 2010.

21. Abdullah, Siti Norul Huda Sheikh, Khairuddin Omar, Shahnorbanun Sahran, and Marzuki Khalid. "License plate recognition based on support vector machine." In 2009 International Conference on Electrical Engineering and Informatics, vol. 1, pp. 78-82. IEEE, 2009.

22. Lekhana, G. C., Srikantaswamy, R. "Real time license plate recognition system", International Journal of Advanced Technology \& Engineering Research 2.4: 5-9, 2012.

23. Lee, Jin-Ki, et al. A Study on Recognition of Both of PCA and LDA Using Types of Vehicle Plate. The Journal of Korea Institute of Information, Electronics, and Communication Technology 6.1: 6-17, 2013.

24. Mingqiang, Yang, Kpalma Kidiyo, and Ronsin Joseph. "A survey of shape feature extraction techniques", Pattern recognition 15.7: 43-90, 2008. 